\title{
Review on Automatic Music Generation System with Deep learning
}

\author{
Prof. Amita Suke, Prof. Khemutai Tighare, Yogeshwari Kamble
}

M. Tech, Department of Computer Science and Engineering, Wainganga College of Engineering and Management, Nagpur, Maharashtra, India

\section{ABSTRACT}

\section{Article Info}

Volume 7, Issue 3

Page Number: 332-335

\section{Publication Issue :}

May-June-2021

\section{Article History}

Accepted : 17 May 2021

Published : 25 May 2021
The music lyrics that we generally listen are human written and no machine involvement is present. Writing music has never been easy task, lot of challenges are involved to write because the music lyrics need to be meaningful and at the same time it needs to be in harmony and synchronised with the music being play over it. They are written by experienced artist who have been writing music lyrics form long time. This project tries to automate music lyrics generation using computerized program and deep learning which we produce lyrics and reduce the load on human skills and may generate new lyrics and a really faster rate than humans ever can. This project will generate the music with the assistance of human and AI

Keywords: Music, Music Generation System, Deep Learning

\section{INTRODUCTION}

The Automatic Music Generation may be a process to make a model by which we are composing a brief piece of music with minimum human intervention. It all started by randomly selecting sounds and mixing them to make a bit of music. Music is essentially composed of Notes and Chords. Deep Learning is a field of Machine Learning which is inspired by a neural network. These networks extract the different features from the dataset related to the object and are capable of learning any nonlinear function, that's why Neural Networks are called as Universal Functional Approximators. Deep Learning are the state of model in various fields like Natural Language Processing (NLP), Computer Vision, Speech Synthesis and so on. Music is a sequence of elements (or sounds) that occurs by chance, hence formulated it using stochastic theory. My random selection of elements which is strictly dependent on mathematical concepts.

I am going to take some existing music data then train a model using this existing data. The prepared model has to learn the patterns in music and its respective lyrics that we humans enjoy, once it learns this, the model should be able to generate new music for us. It cannot simply copy-paste from the training data. It has to understand the patterns/sequence of music to generate new music. I am here not expecting our model to generate new music which is of professional quality, but we want it to generate a decent quality music which should be melodious and good to hear, music is a sequence of musical notes. Our input to the model is a sequence or pattern of musical events/notes. Our output will be 
new sequence/pattern of musical events/notes. In this case-study we have limited to single instrument music as this is our first cut model. In future, this model extend for multiple instrument music.

\section{LITERATURE REVIEW}

Random numbers are fed into model to determine the notes and note lengths of the music with chosen instruments and percussion. This model can generate completely random or musical compositions according to user preferences, even though its aim is not imitating the past genius composers and compose perfectly musical pieces lyrics. The experimental results reflected that this composition model can generate completely random or slightly artistic music having characteristics of Turkish makam music, hence introduce inspiration and entertainment to Turkish tmakam music enthusiasts. The random TMM generation system can be extended to a "random world music" project which may embrace Chinese, Indian, European, American etc.

The context of automated music generation, this sort of knowledge are often of great use to model both the composition itself and its expressive performance. during this paper one computational method to perform this type of research is described. Its implementation is then accustomed generate short musical phrases according to a hierarchical structure that is also used to model the performance of these melodies. The harmonic context is needed to compute two of these weights, thus the score representation used as input for this analysis must include harmonic information.In this implementation MusicXML files that use textual chord annotations over a single melodic line were used, but other solutions are possible: for instance, it would be possible to compute the current chord in a polyphonic MIDI file by analysing all the notes that are being played simultaneously to the considered one. only informal judgements were used as a form of evaluation. For what concerns the generation of melodies, it is clear that the system is very limited in the extent of what can be produced: melodies longer than those shown here tend to result messy. Even in these short excerpts, it is not really clear what is the tonal context, since the algorithm at this stage makes no distinction between major and minor modes, the resulting phrases contain many modulations. Also, many possibilities normally available to composers were not addressed in this first implementation, like for instance anacrusis, pauses, or of course polyphony

\section{PROPOSED METHOD}

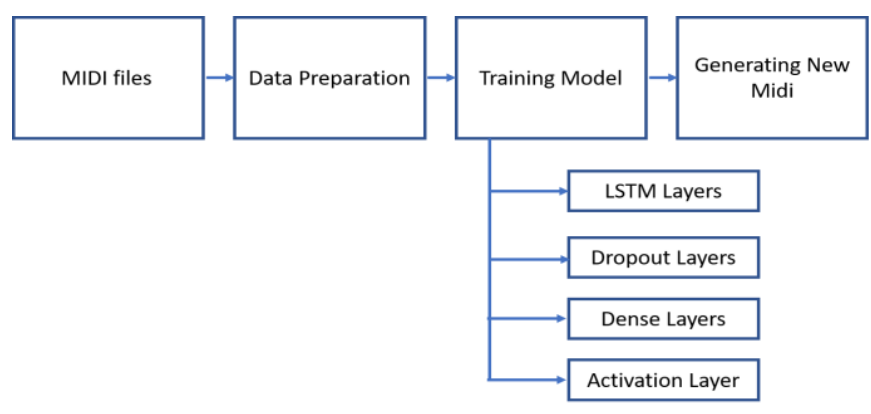

Here MIDI is our data by which I train our model and again I produce another system generated MIDI files. MIDI (an acronym for Musical Instrument Digital Interface) is a computer storage information standard which describes a communication protocol, digital interface and electrical connectors that connect a wide variety of electronic musical device, computer and audio video devices for enjoying, editing and recording music.

\section{Data Preparation:}

Now I am going examined the data which is basically the MIDI file and determined the features that I want to use are the notes and chords as the input and output of our LSTM network. It is time to prepare the data for the neural network. I started by loading each file into a Music21 stream object by using the converter parse(file) function. Using the stream 
object, I get a list of all notes and chords in the file from the parse file function. I append the pitch of every note object using their string object since the most significant parts of the note can be regenerated using the string object of the pitch. And I append every chord by encoding their ID of every note in the chord together into a single string, with each note being separated by a dot. These encodings allow me to easily decode the generated output by the network into the correct notes and chords

\section{Training Model}

Finally, I get to designing the model architecture. In my model I am going to use four different types of layers which are mentioned below:

LSTM layers is a Recurrent Neural Network layer which takes a sequence as an input and can return either sequences (return_sequences=True) or a sequence matrix.

Dropout layers are a regularisation method that consists of setting a fraction of input units to 0 on each update during the training to avoid overfitting. The fraction is determined by the given parameter used with the layer.

Dense layers or fully connected layers is a fully connected neural network layer where each input node is connected to respective output node.

The Activation layer determines what activation function uses our neural network will use to calculate the respective output of a node.

\section{Generating new MIDI files}

To use the neural network to recreate music I will have to put it into the same state as before. For simplicity I will use the same code from the training section as to prepare the data and set up the network model in the same way as before. Except, that instead of training the network I will load the weights which I saved during the training section into the model. Now I can use the trained model to start recreating notes. Since I have a complete list of note sequences at our disposal, we will pick a random index from the list as our starting point, this allows us to rerun the recreation code without changing anything and get different results every iteration. However, if I wish to control the starting point simply instead of the random function, I have to go with a command line argument.

\section{RESULTS AND CONCLUSION}

Now it's time to marvel at the results. Music representation of music that was generated using the LSTM network. At a high level review we can see that there is some structure to it. This is especially obvious within the third to last line on the second page. People that are intimate music and may read notation can see that there are some weird notes strewn about the sheet. This is a results of the neural network not having the ability to make perfect melodies. With our current implementation there'll always be some false notes and to be ready to achieve better results we'll need a much bigger network.

\section{REFERENCES}

[1]. Asst. Ismail Hakkı Parlak, Asst. Cem Kösemen, 2018. Automatic Music Generation by True Random Numbers for Turkish Makams. 2018 4th International Conference on Computer and Technology Applications

[2]. Filippo Carnovalini, Antonio Roda, 2019. A Multilayered Approach to Automatic Music Generation and Expressive Performance. 2019 International Workshop on Multilayer Music Representation and Processing (MMRP)

[3]. Shubham Jain , A. Pandian, 2018. A survey on automatic music generation. International Journal of Engineering \& Technology, 7 (2.8) (2018) 677-679

[4]. Aozhi Liu, Jianzong Wang*, Junqing Peng, Yiwen Wang, Yaqi Mei, Xiaojing Liang, Zimin 
Xia, Jing Xiao, 2019. Composer4Everyone: Automatic Music Generation with Audio Motif. 2019 IEEE Conference on Multimedia Information Processing and Retrieval (MIPR)

[5]. Liao Enyang, Tao Jun,2012. A Research about Automatical Music Generation by Computer. 2012 IEEE Symposium on Robotics and Applications(ISRA)

\section{Cite this article as :}

Prof. Amita Suke, Prof. Khemutai Tighare, Yogeshwari Kamble, "Review on Automatic Music Generation System with Deep learning", International Journal of Scientific Research in Computer Science, Engineering and Information Technology (IJSRCSEIT), ISSN : 2456-3307, Volume 7 Issue 3, pp. 332-335, May-June 2021. Available at doi : https://doi.org/10.32628/CSEIT217340 Journal URL : https://ijsrcseit.com/CSEIT217340 